\title{
Diagnostic significance of haematological testing in patients presenting at the Emergency Department
}

\author{
Giuseppe Lippi*, Giovanni Targher**. Giampaolo Neri***, Clarissa Cattabiani*, Silvia Pipitone*, \\ Gianfranco Cervellin*** \\ * U.O. Diagnostica Ematochimica, Dipartimento di Patologia e Medicina di Laboratorio, Azienda Ospedaliero-Universitaria di \\ Parma \\ ** Sezione di Endocrinologia e Metabolismo, Dipartimento di Medicina, Università degli Studi di Verona \\ *** U.O. Pronto Soccorso e Medicina d'Urgenza, Dipartimento di Emergenza-Urgenza, Azienda Ospedaliero-Universitaria di \\ Parma
}

Key words: emergency department, haematological testing, lavoratory testing, rule-out test, diagnosis.

\section{Abstract}

The use of simple and economic tests to rule out diseases of sufficient clinical severity is appealing in emergency department (ED), since it would be effective for contrasting ED overcrowding and decreasing healthcare costs. The aim of this study was to assess the diagnostic performance of simple and economic haematological testing in a large sample of adult patients presenting at the ED of the Academic Hospital of Parma during the year 2010 with the five most frequent acute pathologies (i.e., acute myocardial infarction, renal colic, pneumonia, trauma and pancreatitis). Both leukocyte count and hemoglobin showed a good diagnostic performance (Area Under the Curve [AUC] of 0.85 for leukocyte count and 0.76 for hemoglobin; both $\mathrm{p}<0.01)$. Although the platelet count was significantly increased in all patients groups except pancreatitis, the diagnostic performance did not achieve statistical significance (AUC 0.53; $\mathrm{p}=0.07$ ). We also observed an increased RDW in all groups, except in those with trauma and the diagnostic performance was acceptable (AUC 0.705; $<<0.01$ ). The mean platelet volume (MPV) was consistently lower in all patients groups and also characterized by an efficient diagnostic performance (AUC 0.76; $\mathrm{p}<0.01$ ). This evidence led us to design an arbitrary formula, whereby MPV and hemoglobin were multiplied, and further divided by the leukocyte count, obtaining a remarkable AUC $(0.91 ; \mathrm{p}<0.01)$. We conclude that simple, rapid and cheap hematological tests might provide relevant clinical information for decision making to busy emergency physicians, and the their combination into an arbitrary formula might further increase the specific diagnostic potential of each of them.

\section{Introduction}

Hospital-based Emergency Departments (EDs) evolved during the past two decades to provide not only acute emergency care but also safety net care for indigent patients, public health surveillance, disaster preparedness, observation and procedural care, occupational care, employee health, and, in many cases, primary health care. Despite these expanding services, the ED resources, including overall number of ED facilities, beds within those facilities as well as funding, did not increase proportionally.

The overcrowding in EDs and its potentially adverse consequences, is a well-known phenomenon in Western countries (1). Multiple factors are likely to be involved in the growing crisis of ED crowding. Among them, in our country, there is the constantly decreasing number of hospital beds, the steadily increasing number of ED visits and the demographic changes of the population (i.e. increasing prevalences of elderly people and migrants). ED overcrowding accounts different timing of various care-paths (e.g. door-to-balloon time in STEMI) and may also influence the degree of care given to patients (1). ED overcrowding has also a variety of other undesirable consequences, including increased patient waiting times, decreased ability to protect patient privacy and confidentiality, impaired evaluation and treatment, and difficulties in delivering personcentered care. These consequences can be understood not just as undesirable or unfortunate in terms of obvious operational and logistic problems for hospitals but also as violations of widely held, fundamental, ethical norms (2). The ED overcrowding has also been considered as a first-line cause of doctors' and nurses' burnout and of verbal or physical assault. Overall, there is an emerging body of evidence suggesting that ED overcrowding is more effectively influenced by interventions mainly directed to improve patient's output from ED to wards; by contrast, interventions mainly directed to reduce the amount of ED visits failed to be effective. One reliable approach for contrasting ED overcrowding may be represented by the use of simple and economical laboratory tests to enhance the efficiency of triage. Diagnostics investigations, including laboratory testing, are typically subjected to a Bayesian approach and thereby are characterized by two leading characteristics, i.e. sensitivity and specificity, which contribute to define the negative and positive predictive value (i.e. NPV and PPV) as well as the positive and negative likelihood ratio (i.e. LR+ and LR-) (3). The use of simple and economic tests to rule out rather than diagnose diseases of sufficient clinical severity -the so-called "negative thinking" - is hence appealing in EDs, since it would be effective for contrasting ED overcrowding as well as for decreasing healthcare costs. Thus, the main aim of the present study was to assess the diagnostic significance and performance of simple and economic haematological testing in a large sample of adult patients presenting at the ED of the Academic Hospital of Parma with acute diseases and requiring a fast triage. 


\section{Material and Methods}

We selected the five most frequent acute pathologies (i.e. acute myocardial infarction, renal colic, pneumonia, trauma and pancreatitis) which occurred in adult patients who were acutely admitted to the ED of the Parma University Hospital during the year 2010. All the hospital records of adult patients who were consecutively visited in our ED from January 1 th, 2010 to December 31th, 2010 were extracted from our informatic database. The selection of index cases followed the diagnostic criteria universally accepted for each specific pathology and the trauma cases included those with Injury Severity Score (ISS) $>15$. Out of the total number of patients identified for each disease, $25 \%$ of total cases have been extracted with a program of randomization to preserve the homogeneity of the sample population and quickening the statistical analysis of data.

The clinical and haematological data were firstly retrieved using both the ICD-9 code and the related diagnostic terms (i.e. "myocardial infarction", "renal colic", "pneumonia", "trauma" and "pancreatitis") and were subsequently reviewed for deleting wrong registrations. The final sample consisted of 462 acutely admitted ED patients (i.e. cases) who had the following definitive diagnoses: 262 renal colic, 62 pneumonia, 51 acute pancreatitis, 46 acute myocardial infarction (AMI) and 41 trauma. We also retrieved the demographic and haematological data from a healthy control population, which consisted of 429 blood donors (i.e. controls), who were matched for age and sex to the cases, and who referred to our hospital for a regular blood donation.

In both controls and cases, haematological testing were performed on the same laboratory instrumentation (i.e. Sysmex XE 2100, Sysmex Inc, Mundelein IL, USA). Among the various haematological parameters, we arbitrarily selected those which were more frequently informative in urgent testing such as hemoglobin, white blood cell (WBC), platelet (PLT) counts, as well as mean platelet volume (MPV) and red blood cell distribution width (RDW), i.e. two simple parameters that have been recently shown to be significantly associated with acute diseases. The quality of laboratory results have been validated throughout the study period by regular internal quality control (IQC) procedures and participation in an External Quality Assessment Scheme (EQAS).

Data were presented as means \pm standard deviation (SD) or proportions. Skewed variables were logarithmically transformed to improve normality prior to analyses. The significance of differences between the groups was assessed by the one-way analysis of variance (for continuous variables) and the chi-squared test with Yates's correction for continuity (for categorical variables). Univariate linear regression analysis was also performed. Diagnostic performance of the haematological tests was evaluated by the Receiver Operating Characteristic (ROC) curve analysis. P-values $<0.05$ were considered statistically significant.

\section{Results}

\section{Values of haematological parameters among the different pathological conditions}

The main demographic and haematological characteristics of healthy controls and acutely admitted ED patients (considered as either a whole sample or subdivided according to their acute pathologies) are summarized in Table 1. By study design, no significant differences were found in age and gender distribution between the control group and the patients' group, except for those with acute pancreatitis, who were more likely to be older than healthy controls. As regards the haematological parameters, significant differences were found for hemoglobin concentration (i.e., hemoglobin was lower in all cases, irrespective of different acute pathologies, than in controls), WBC count (i.e., WBC was higher in all cases, irrespective of different pathologies) and MPV (i.e., mean MPV was lower in all cases irrespective of different pathologies). PLT count was also higher in all cases as well as in various disease groups, except in those with pancreatitis. Similarly, RDW was higher in all cases as well as in AMI, pancreatitis and pneumonia groups, but it was lower in the renal colic group and non-significantly different in the trauma group.

A significant, inverse, association was observed between MPV and PLT in healthy controls $(r=-0.340 ; p<0.01)$ and in all cases $(r=-0.306 ; p<0.01)$ as well as in the renal colic $(r=-0.330 ; p<0.01)$, AMI $(r=-0.485 ; p<0.01)$, pneumonia $(r$ $=-0.392 ; \mathrm{p}<0.01)$ and trauma $(\mathrm{r}=-0.317 ; \mathrm{p}=0.01)$ groups, but not in the pancreatitis group $(\mathrm{r} p=-0.183 ; \mathrm{p}=0.199)$.

\section{ROC curve analysis}

The results of ROC curve analyses for hemoglobin, RDW, WBC, MPV and PLT are shown in Table 2. When comparing healthy controls versus cases, there were significant AUC values for hemoglobin (AUC 0.71; p < 0.01), MPV (AUC 0.76; $\mathrm{p}<0.01$ ) and WBC (AUC 0.85; $<<0.01$ ), but not for PLT count and RDW.

As regards the different acute pathologies, the AUC of hemoglobin was statistically significant in all instances, except for patients with pancreatitis versus all other pathological groups. The AUC of RDW was significant when comparing renal colics versus all other pathological groups, AMI versus controls, pancreatitis versus controls, pneumonia versus controls, and pneumonia versus all other pathological groups. The AUC of MPV was significant in all instances, except for AMI patients versus all other pathological groups, pancreatitis versus all other pathological groups, pneumonia versus all other pathological groups, and trauma versus all other pathological groups. The AUC of WBC was significant in all instances, except for AMI patients versus all other pathological groups, pancreatitis versus all other pathological groups and pneumonia versus all other pathological groups. Conversely, the AUC of PLT did not achieve a statistical significance in any case. 
According to thes above-mentioned results, we then decided to combine the most informative haematological parameters, i.e. hemoglobin, MPV and WBC count, in an arbitrary formula based on three parameters and derived from a single test (i.e., complete blood cell count; [hemoglobin x MPV] / WBC). The relative values were substantially different between healthy controls and cases (as shown in Table 1 and Figure 1).

Table 1. Main demographic and hematological characteristics of the study population.

\begin{tabular}{|c|c|c|c|c|c|c|c|}
\hline & $\begin{array}{l}\text { Healthy } \\
\text { Controls }\end{array}$ & All cases & Renal colic & $\begin{array}{l}\text { Myocardial } \\
\text { Infarction }\end{array}$ & Pancreatitis & Pneumonia & Trauma \\
\hline $\mathrm{n}$ & 429 & 462 & 262 & 46 & 51 & 62 & 41 \\
\hline Age (years) & $48 \pm 16$ & $49 \pm 17$ & $48 \pm 18$ & $47 \pm 9$ & $56 \pm 14 \ddagger$ & $46 \pm 15$ & $44 \pm 18$ \\
\hline Gender (M/F) & $241 / 188$ & $245 / 217$ & $134 / 128$ & $29 / 17$ & $24 / 27$ & $33 / 29$ & $16 / 25$ \\
\hline Hemoglobin (g/L) & $148 \pm 12$ & $136 \pm 19 \ddagger$ & $140 \pm 16$ 丰 & $130 \pm 24 \neq$ & $137 \pm 19$ \# & $126 \pm 20 \ddagger$ & $133 \pm 15 \ddagger$ \\
\hline $\mathrm{WBC}\left(10^{9} / \mathrm{L}\right)$ & $6.2 \pm 1.4$ & $9.7 \pm 3.5 \neq$ & $9.2 \pm 2.9 \ddagger$ & $10.0 \pm 4.3 \ddagger$ & $10.4 \pm 3.7 \neq$ & $10.6 \pm 4.5 \neq$ & $12.7 \pm 4.6$ 丰 \\
\hline $\operatorname{PLT}\left(10^{9} / \mathrm{L}\right)$ & $231 \pm 48$ & $241 \pm 71 \neq$ & $242 \pm 64 \neq$ & $243 \pm 62$ 丮 & $233 \pm 80$ & $243 \pm 86 \dagger$ & $250 \pm 12 \dagger$ \\
\hline RDW (\%) & $13.5 \pm 0.7$ & $13.8 \pm 1.8 \neq$ & $13.1 \pm 1.0 \dagger$ & $14.6 \pm 2.7 \ddagger$ & $14.3 \pm 2.1 \neq$ & $14.8 \pm 2.7$ 丰 & $13.5 \pm 0.9$ \\
\hline MPV (fL) & $11.1 \pm 0.9$ & $10.1 \pm 1.2$ 丰 & $10.0 \pm 1.2 \neq$ & $10.3 \pm 1.1 \neq$ & $10.6 \pm 1.2 \dagger$ & $10.1 \pm 1.1$ 丰 & $10.4 \pm 0.9 \neq$ \\
\hline Formula* & $277 \pm 64$ & $160 \pm 68 \mp$ & $168 \pm 62 \ddagger$ & $156 \pm 67$ \# & $151 \pm 62 \neq$ & $143 \pm 89 \neq$ & $123 \pm 46 \ddagger$ \\
\hline
\end{tabular}

Data are presented as means \pm SD or proportions.

PLT, Platelet count; WBC, White Blood Cell count; MPV, Mean Platelet Volume; RDW, Red Blood Cell Distribution Width.

* Formula: [hemoglobin x MPV] / WBC count

$\dagger \mathrm{p}<0.05$; $\neq \mathrm{p}<0.01$ for differences versus healthy controls

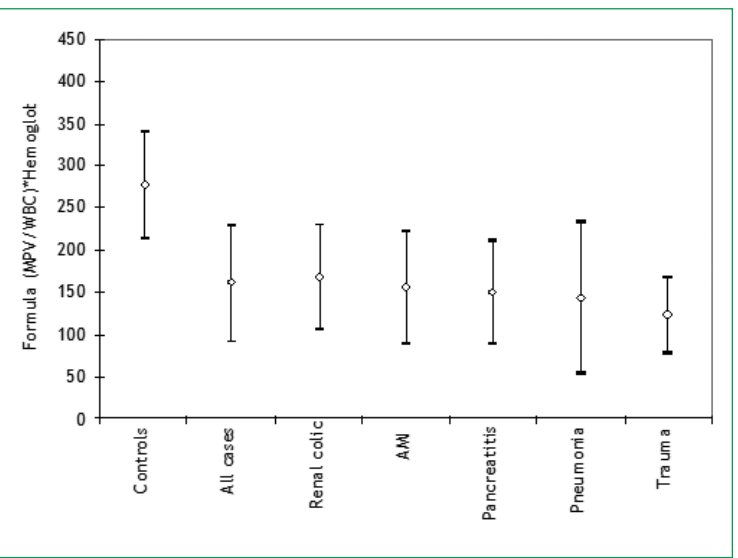

Figure 1. Distribution values (expressed as mean \pm standard deviation) of the arbitrary formula (calculated as [hemoglobin $\mathrm{x}$ MPV] / WBC) in 429 healthy controls and in 462 patients, who were admitted to the Emergency Department for various acute diseases.

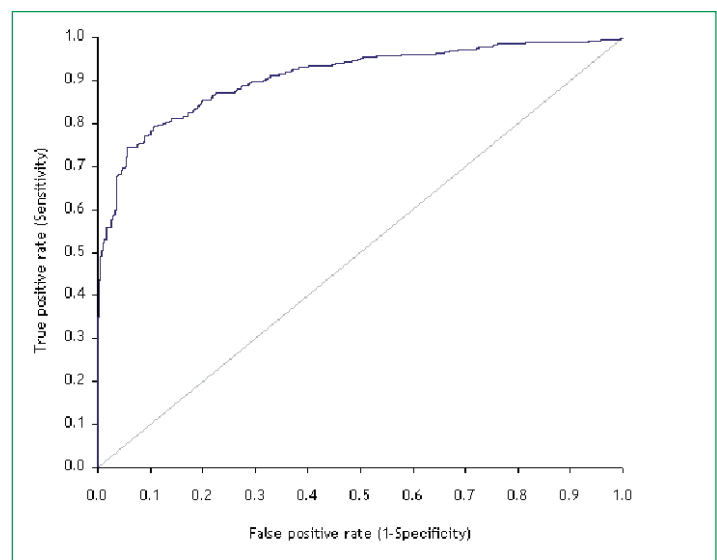

Figure 2. Area under the ROC curve analysis of the arbitrary formula (calculated as [hemoglobin $\mathrm{x}$ Mean Platelet Volume] / leukocyte count) for identifying patients with acute pathologies and healthy controls (Area Under the Curve: AUC 0.91; $<<0.01$ ).

Interestingly, we observed a good overall performance of this formula for distinguishing between healthy controls from patients, i.e., AUC 0.91 ( $\mathrm{p}<0.01$ ) (Figure 2). The best diagnostic performance of this formula was obtained for an arbitrary cut-off value of 220.5 , which corresponds to an $85 \%$ sensitivity, $84 \%$ NPV, 0.18 LR-, $80 \%$ specificity, $81 \%$ PPV and 4.25 LR+. A cut-off value of 274.6 was instead associated with high values of sensitivity (95\%), NPV (91\%) and LR- (0.1), combined with an acceptable specificity (i.e. 50\%), PPV (66\%) and LR+ (1.9). Remarkable diagnostic performances of this formula were also obtained when comparing the control group with the groups of those with renal colic (AUC 0.89; $<<$ 0.01), AMI (AUC 0.91; p < 0.01), pancreatitis (AUC 0.91; p < 0.01), pneumonia (AUC 0.93; p < 0.01) or trauma (AUC 0.97; $\mathrm{p}<0.01$ ). As regards the comparison among the different patient groups, this formula also enabled to significantly distinguish the renal colic group from all other pathological groups (AUC 0.64; $p<0.01$ ), the pneumonia group from all other pathological groups (AUC $0.62 ; \mathrm{p}=0.01$ ) and the trauma group from all other pathological groups $(\mathrm{AUC}=0.69 ; \mathrm{p}$ 
$<0.01$ ), respectively, but not the AMI and the pancreatitis groups from all other pathological groups (AUC 0.50; $\mathrm{p}=0.49$ and AUC 0.55; p = 0.15, respectively). However, the results obtained in these subgroups analyses should be interpreted with some degree of caution given the relatively low number of patients included.

\section{Discussion}

Nearly half of the patients presenting at the ED have often non-urgent conditions, which might be safely referred elsewhere, either to other healthcare settings or discharged (4). This can only be accomplished by using fast, simple and economical tests, which should also be characterized by efficient sensitivity, NPV and LR-. The conventional haematological testing provides a variety of parameters, which may also be useful for the diagnostic reasoning of several non-hematological pathologies. Among the various parameters that are provided by the modern haematological analyzers, those most informative for acute pathologies as well as those more frequently consulted by emergency physicians are indeed the hemoglobin concentration, WBC and PLT counts. The hemoglobin value, more than the hematocrit and the RBC count, provides information about the presence of anaemia, which is defined as a hemoglobin concentration lower than $120 \mathrm{~g} / \mathrm{L}$ in women and lower than $135 \mathrm{~g} / \mathrm{L}$ in men (5). The WBC count helps identify leukopenia or leukocytosis. By definition, leukocytosis (frequently arising from relatively benign conditions such as infectious diseases or inflammatory processes as well as from serious causes including primary bone marrow disorders) and leukopenia (mainly due to bone marrow failure) in the adulthood are diagnosed when WBC count is increased to more than $10 \mathrm{x} 10^{9} / \mathrm{L}$, or decreased to less than $4.0 \times 10^{9} / \mathrm{L}$, respectively (6). The PLT count, whose reference range is comprised between 150 and $400 \times 10^{9} / \mathrm{L}$, is instead useful for identifying thrombocytopenia (caused by either decreased production or increased consumption in the bloodstream) and thrombocytosis (either due to myeloproliferative disorders or reactive causes) (7).

Besides these very common tests, additional well-known hematological parameters, especially RDW and MPV, are now experimenting an important "resurrection" in the diagnostic approach to several non-hematological disorders. The RDW expresses the variation in the size of red cells (anisocytosis). Its normal values are typically comprised between 11.5 and $14.5 \%$ (8). Beside the undeniable usefulness for the initial classification of anaemia in the ED (9), some recent studies have evaluated the role of RDW in the diagnostic approach to patients with AMI. In a previous investigation, we found that a higher RDW value was significantly associated with an increased odds of AMI, showing an overall AUC of 0.705 ( $p<0.01$ ) (10). In a subsequent investigation, using a different instrumentation, we failed to find any significant difference in RDW values between AMI patients and controls (11). However, a higher RDW value was found to be strongly and independently associated with an increased risk of incident AMI and death in some large prospective studies (12-15). With regard to other pathologies, increased RDW values were observed in patients with community acquired pneumonia (16), ischemic stroke (17), chronic kidney disease (18), thrombotic thrombocytopenic purpura (19) or coeliac disease (20). A significant, graded relationship was also found between higher RDW and inflammatory biomarkers, namely highly sensitive C-reactive protein and erythrocyte sedimentation rate, in a large cohort of unselected adult outpatients (18). To our knowledge, no data are currently available about the association of RDW with other acute pathologies that are commonly observed at the ED, such as renal colic, trauma and pancreatitis. The MPV expresses the average size of platelets in blood. The typical range of platelet volumes is $9.7-12.8 \mathrm{fL}$, which is roughly equivalent to 2.65 to $2.9 \mu \mathrm{m}$. Increased MPV has usually been considered as a reliable index of larger, more reactive, platelets often resulting from an increased PLT turnover. As such, increased MPV has been observed in patients with AMI (21-23), hypertension (24), sepsis (25), dysglycemia (26), non-alcoholic fatty liver disease (27), hypothyroidism (28) and chronic spontaneous urticaria (29). Conversely, a decreased MPV has been observed in patients with persistent bacterial infections (30). Regarding the results of our case-control study, leukocytosis and anaemia were commonplace throughout the five groups of acutely admitted ED patients (Table 1). Both WBC count and hemoglobin were characterized by a good diagnostic performance, as attested by the AUC of ROC curve analyses (all cases versus controls: AUC 0.85 and 0.76 for WBC count and hemoglobin, respectively; both $\mathrm{p}<0.01$ ) (Table 2). The PLT count was also significantly increased in all patients groups, except in those with pancreatitis. Nevertheless, a substantial overlap in PLT distribution values was observed and, accordingly, in no instance the AUC of this parameter achieved a statistical significance (Table 2). In agreement with previously published studies, we observed an increased RDW value in all groups of sdmitted ED patients, except in those with trauma, confirming that this parameter would mirror the presence of anisocytosis that might aspecifically occur throughout a variety of acute disorders. Nevertheless, the rather broad distribution and the substantial overlap of RDW values between cases and controls consistently decreased the diagnostic performance of RDW (all cases versus controls: AUC 0.23; $\mathrm{p}=0.34$ ) (Table 2). Our finding of lower MPV values in all patients groups than in controls apparently disagrees with previous observations, inasmuch as a higher MPV value was described in a variety of disorders. A reliable explanation however may exist. The platelets undergo at least two patterns of reactive size changes, i.e. an early rise, which is associated with thrombocytopenia, and a later fall, which is often associated with thrombocytosis (30). In our investigation, the mean PLT value was significantly higher in all acute disease groups than in the control group, except in those with pancreatitis. Even more importantly, an inverse association between PLT count and MPV could be observed in all patient groups, except in those with pancreatitis. It is thereby conceivable that the lower MPV value observed in our acutely admitted ED patients would reflect the latter phase of reactive size change (i.e. reduction), which has been described to occur in association with thrombocytosis. Accordingly, patients with pancreatitis had the highest value of MPV as well as the lowest PLT count. Interestingly, MPV was characterized by an efficient diagnostic performance (all cases versus controls: AUC 0.76 ; p < 0.01 ). The two evidences of significant AUC values for WBC count, MPV and hemoglobin, along with the value distributions in 
the acute disease groups (increased values of WBC count, decreased values of hemoglobin and MPV), prompted us to design an arbitrary formula, whereby MPV and hemoglobin values were multiplied, and further divided by the WBC count. The highest the value of this formula, the lowest the probability of diagnosing one of the five acute diseases investigated in this study, as shown in Figure 1. The AUC of this formula was remarkable; the choice of a 274.6 cut-off value would have yielded an optimal performance for rapid rule out of all of the five acute pathologies investigated in our study.

The are two leading take-home messages from this case-control study. First, we demonstrated that three parameters (i.e., MPV, Hb and WBC) deriving from simple, rapid and cheap laboratory tests, such as those attainable from routine hematological testing, show optimal diagnostic performance and might provide relevant clinical information for decisionmaking to busy emergency physicians. Second, we demonstrated that the combination of three of these simple laboratory tests within an arbitrary formula might consistently increase the diagnostic potential of each of them when considered separately. It is however noteworthy that the arbitrary formula which we developed was derived from a local database of acutely admitted ED patients, and that the results were obtained using a single specific laboratory instrumentation. As such, the clinical usefulness of this formula needs to be further validated in other clinical settings and using different hematological analyzers. Nevertheless, we believe that this finding represents a paradigmatic example, which can be assessed in further larger investigation, to establish whether either different threshold values of this proposed formula or different combination of simple and inexpensive haematological parameters could help the clinical decision-making of physicians in acutely admitted ED patients. In synthesis, these findings represent a reliable example on how easy and inexpensive parameters or indices such as those proposed in this study can be incorporated within a more comprehensive approach to risk stratification of patients acutely admitted to the ED. A very simple parameter such as the WBC, for example, does not confirm or rule out any pathology alone, but it can be included in some steps of the diagnostic reasoning along with other clinical and laboratory parameters, where it can instead provide meaningful clinical information.

Table 2. Diagnostic performances of different haematological parameters in 429 healthy controls and in 462 patients who were admitted to the Emergency Department for various acute pathologies.

\begin{tabular}{|l|l|l|l|l|l|l|l|l|l|l|l|l|l|}
\hline & PLT & & \multicolumn{2}{|l|}{ Hemoglobin } & \multicolumn{2}{l|}{ RDW } & \multicolumn{2}{l|}{ MPV } & \multicolumn{2}{l|}{ WBC } \\
\hline & AUC & P & AUC & P & AUC & P & AUC & P & AUC & P \\
\hline $\begin{array}{l}\text { All cases versus } \\
\text { controls }\end{array}$ & 0.53 & 0.07 & 0.76 & $<0.01$ & 0.71 & $<0.01$ & 0.76 & $<0.01$ & 0.85 & $<0.01$ \\
\hline $\begin{array}{l}\text { Renal colics } \\
\text { versus controls }\end{array}$ & 0.54 & 0.06 & 0.67 & $<0.01$ & 0.51 & 0.89 & 0.78 & $<0.01$ & 0.83 & $<0.01$ \\
\hline $\begin{array}{l}\text { Renal colics } \\
\text { versus all cases }\end{array}$ & 0.52 & 0.41 & 0.61 & $<0.01$ & 0.65 & $<0.01$ & 0.55 & 0.03 & 0.61 & $<0.01$ \\
\hline $\begin{array}{l}\text { AMI versus } \\
\text { controls }\end{array}$ & 0.55 & 0.86 & 0.75 & $<0.01$ & 0.66 & $<0.01$ & 0.72 & $<0.01$ & 0.84 & $<0.01$ \\
\hline $\begin{array}{l}\text { AMI versus all } \\
\text { cases }\end{array}$ & 0.52 & 0.71 & 0.55 & $<0.01$ & 0.63 & 0.04 & 0.51 & 0.41 & 0.53 & 0.29 \\
\hline $\begin{array}{l}\text { Pancreatitis } \\
\text { versus controls }\end{array}$ & 0.53 & 0.15 & 0.69 & $<0.01$ & 0.60 & 0.02 & 0.73 & $<0.01$ & 0.86 & $<0.01$ \\
\hline $\begin{array}{l}\text { Pancreatitis } \\
\text { versus all cases }\end{array}$ & 0.56 & 0.06 & 0.53 & 0.08 & 0.59 & 0.01 & 0.53 & 0.24 & 0.45 & 0.87 \\
\hline $\begin{array}{l}\text { Pneumonia } \\
\text { versus controls }\end{array}$ & 0.51 & 0.49 & 0.83 & $<0.01$ & 0.64 & $<0.01$ & 0.77 & $<0.01$ & 0.78 & $<0.01$ \\
\hline $\begin{array}{l}\text { Pneumonia } \\
\text { versus all cases }\end{array}$ & 0.52 & 0.38 & 0.58 & 0.01 & 0.59 & 0.01 & 0.49 & 0.56 & 0.56 & 0.07 \\
\hline $\begin{array}{l}\text { Trauma versus } \\
\text { controls }\end{array}$ & 0.57 & 0.10 & 0.80 & $<0.01$ & 0.45 & 0.84 & 0.72 & $<0.01$ & 0.94 & $<0.01$ \\
\hline $\begin{array}{l}\text { Trauma versus } \\
\text { all cases }\end{array}$ & 0.51 & 0.76 & 0.54 & 0.03 & 0.53 & 0.25 & 0.57 & 0.06 & 0.71 & $<0.01$ \\
\hline
\end{tabular}

Data are presented as area under the ROC curve (AUC)

PLT, Platelet count; WBC, White Blood Cell count; MPV, Mean Platelet Volume; AMI, Acute Myocardial Infarction; RDW, Red Blood Cell Distribution Width.

Important limitations of this study are that the significance of haematological testing was only assessed in five acute pathologies, and that we have enrolled healthy blood donors as the control population, whereby patients afflicted by similar symptoms but different final diagnosis might have been more informative in this setting. It is however noteworthy that, taken together, renal colic, AMI, pancreatitis, pneumonia and trauma cover the vast majority of acute causes for ED access in our facility (i.e., more than $50 \%$ of high priority - yellow and red codes). Moreover, the use of healthy blood donors is currently considered the gold standard for establishing whether a biochemical test is helpful or not for 
distinguishing between health and disease. Another potential limitation of this study is the high prevalence of patients with renal colic, which represented nearly half the sample population and might have thereby somehow affected the outcome of the study.

\section{References}

1. Rastelli G, Cavazza M, Cervellin G. Sovraffollamento in Pronto Soccorso. Analisi del fenomeno e proposte di gestione. Emerg Care J 2010; 2: 25-35.

2. Agrawal S. Emergency Department crowding: an ethical perspective. Acad Emerg Med 2007; 14: $750-751$.

3. Lippi G, Guidi GC. The power of negative thinking. Am J Emerg Med 2008; 26: 373-374.

4. Bernstein SL, Bernstein E, Boudreaux ED, Babcock-Irvin C, Mello MJ, Kapur AK et al. Public health considerations in knowledge translation in the Emergency Department. Acad Emerg Med 2007; 14: 1036-1041.

5. World Health Organization. Worldwide prevalence of anaemia 1993-2005. WHO Press, Geneva, 2008.

6. Munker R. Leukocytosis, Leukopenia, and Other Reactive Changes of Myelopoiesis, in Munker R, Hiller E, Glass J, Paquette R eds. Modern Hematology: Biology and Clinical Management. Humana Press-Springer, New York, 2007.

7. Schmaier AH. Laboratory evaluation of hemostatic and thrombotic disorders, in Hoffman R, Benz EJ Jr, Shattil SJ et al, eds. Hoffman Hematology: Basic Principles and Practice. Churchill Livingstone Elsevier, Philadelphia, Pa, 2008.

8. Sarma PR. Red Cell Indices, in Walker HK, Hall WD, Hurst JW eds. Clinical Methods: The History, Physical, and Laboratory Examinations. Butterworths, Boston, 1990.

9. Evans TC, Jehle D. The red blood cell distribution width. J Emerg Med 1991; 9 (suppl. 1): 71-4.

10. Lippi G, Filippozzi L, Montagnana M, Luca Salvagno G, Franchini M, Cesare Guidi G et al. Short Communication: Clinical usefulness of measuring red blood cell distribution width on admission in patients with acute coronary syndromes. Clin Chem Lab Med 2009; 47: 353-7.

11. Cemin R, Donazzan L, Lippi G, Clari F, Daves M. Blood cells characteristics as determinants of acute myocardial infarction. Clin Chem Lab Med 2011; 49: 1231-6.

12. Zalawadiya SK, Veeranna V, Niraj A, Pradhan J, Afonso L. Red cell distribution width and risk of coronary heart disease events. Am J Cardiol 2010; 106: 988-93.

13. Dabbah S, Hammerman H, Markiewicz W, Aronson D. Relation between red cell distribution width and clinical outcomes after acute myocardial infarction. Am J Cardiol 2010; 105: 312-7.

14. Patel KV, Semba RD, Ferrucci L, Newman AB, Fried LP, Wallace RB et al. Red cell distribution width and mortality in older adults: a meta-analysis. J Gerontol A Biol Sci Med Sci 2010; 65: 258-65.

15. Perlstein TS, Weuve J, Pfeffer MA, Beckman JA. Red blood cell distribution width and mortality risk in a community-based prospective cohort. Arch Intern Med 2009; 169: 588-94.

16. Braun E, Domany E, Kenig Y, Mazor Y, Makhoul BF, Azzam ZS. Elevated red cell distribution width predicts poor outcome in young patients with community-acquired pneumonia. Crit Care 2011; 15: R194.

17. Ani C, Ovbiagele B. Elevated red blood cell distribution width predicts mortality in persons with known stroke. J Neurol Sci 2009; 277: 103-8.

18. Lippi G, Targher G, Montagnana M, Salvagno GL, Zoppini G, Guidi GC. Relationship between red blood cell distribution width and kidney function tests in a large cohort of unselected outpatients. Scand J Clin Lab Invest 2008; 68: 745-8.

19. Nagajothi N, Braverman A. Elevated red cell distribution width in the diagnosis of thrombotic thrombocytopenic purpura in patients presenting with anemia and thrombocytopenia. South Med J 2007; 100: 257-9.

20. Sategna Guidetti C, Scaglione N, Martini S. Red cell distribution width as a marker of coeliac disease: a prospective study. Eur J Gastroenterol Hepatol 2002; 14: 177-81.

21. Endler G, Klimesch A, Sunder-Plassmann H, Schillinger M, Exner M, Mannhalter C et al. Mean platelet volume is an independent risk factor for myocardial infarction but not for coronary artery disease. Br J Haematol 2002; 117: 399-404.

22. Lippi G, Filippozzi L, Salvagno GL, Montagnana M, Franchini M, Guidi GC et al. Increased mean platelet volume in patients with acute coronary syndromes. Arch Pathol Lab Med 2009; 133: 1441-3.

23. Klovaite J, Benn M, Yazdanyar S, Nordestgaard BG. High platelet volume and increased risk of myocardial infarction: 39,531 participants from the general population. J Thromb Haemost 2011; 9: 49-56.

24. Ntaios G, Gurer O, Faouzi M, Aubert C, Michel P. Hypertension is an indepedent predictor of mean platelet volume in patients with acute ischemic stroke. Intern Med J 2010 Jan 4. [Epub ahead of print].

25. Van der Lelie J, Von dem Borne AK. Increased mean platelet volume in septicaemia. J Clin Pathol 1983; 36: 693-6.

26. Zuberi BF, Akhtar N, Afsar S. Comparison of mean platelet volume in patients with diabetes mellitus, impaired fasting glucose and non-diabetic subjects. Singapore Med J 2008; 49: 114-6.

27. Shin WY, Jung DH, Shim JY, Lee HR. The association between non-alcoholic hepatic steatosis and mean platelet volume in an obese Korean population. Platelets 2011; 22: 442-6.

28. Yilmaz H, Erturul O, Erturul B, Erturul D. Mean platelet volume in patients with subclinical hypothyroidism. Platelets $2011 ; 22: 143-7$.

29. Magen E, Mishal J, Zeldin Y, Feldman V, Kidon M, Schlesinger M, Sthoeger Z. Increased mean platelet volume and C-reactive protein levels in patients with chronic urticaria with a positive autologous serum skin test. Am J Med Sci 2010; 339: 504-8.

30. Robbins G, Barnard DL. Mean platelet volume changes in infection. J Clin Pathol 1983; $36: 1320$. 